\title{
A 6-month, multicenter, open-label study of fixed dose naproxen/esomeprazole in adolescent patients with juvenile idiopathic arthritis
}

Daniel J. Lovell ${ }^{1,2}$, Jason A. Dare ${ }^{3}$, Megan Francis-Sedlak ${ }^{4}$, Julie Ball ${ }^{4}$, Brian D. LaMoreaux ${ }^{4}$, Emily Von Scheven ${ }^{5}$, Adam Reinhardt ${ }^{6}$, Rita Jerath ${ }^{7}$, Oral Alpan ${ }^{8}$, Ramesh Gupta ${ }^{9}$, Donald Goldsmith ${ }^{10}$, Andrew Zeft ${ }^{11}$, Henry Naddaf ${ }^{12}$, Beth Gottlieb ${ }^{13}$, Lawrence Jung ${ }^{14}$ and Robert J. Holt ${ }^{4,15^{*}}$ (D)

\begin{abstract}
Background: Juvenile idiopathic arthritis (JIA) is an inflammatory arthritis of unknown etiology, which lasts for greater than 6 weeks with onset before 16 years of age. JIA is the most common chronic rheumatic disease in children. NSAIDs have been the mainstay of initial management with naproxen (NAP) being commonly used, but they may cause serious side effects such as gastric ulcers which can be reduced by concomitant administration of proton pump inhibitors, such as esomeprazole (ESO).
\end{abstract}

Methods: Primary objective was to evaluate the safety and tolerability of 3 fixed doses of NAP/ESO in JIA patients aged 12 to 16 years. Forty-six children and adolescents with JIA by International League of Associations for Rheumatology criteria, mean age of 13.6 years, from 18 US sites were prospectively enrolled over 2 years and followed for up to 6 months. Doses of the NAP/ESO fixed combination were based on baseline weight. The exploratory efficacy outcome was assessed with the ACR Pediatric-30, - 50, - 70, - 90 Response and the Childhood Health Assessment Questionnaire (CHAQ) discomfort and functional scores at months 1, 3, and 6 as change from baseline. Occurrence and causality were assessed for treatment emergent AEs (TEAEs) and discontinuations were monitored monthly.

Results: Forty-six patients received at least 1 dose of naproxen/esomeprazole and 36 completed the trial. Thirty-seven (80.4\%) had at least 1 treatment emergent adverse event (TEAE) and, with the exception of 2 events in one patient, all of the TEAEs were mild or moderate. Frequent TEAEs ( $\geq 5 \%$ of patients) were upper respiratory tract and gastrointestinal related. Eleven (23.9\%) had at least 1 TEAE considered to be related to study drug. Four patients (8.7\%) discontinued due to a TEAE with one of these being the only serious AE reported, acute hepatitis. Mean number of active joints at baseline was 3.1. Improvement in JIA signs and symptoms occurred at most assessments and by month 6 , the percentage of patients with an ACR Pediatric-30, - 50, - 70, and - 90 Response was 47.1, 38.2, 32.4, and 17.6\%, respectively. The percent of patients achieving ACR Pediatric response increased over time. CHAQ discomfort improved at each assessment and functional scores improved at all assessments for 'Arising, Walking, and Activities' with several improved for 'Dressing and Grooming, Eating, Hygiene, and Grip'. There was no indication of a dose-related efficacy effect.

(Continued on next page)

\footnotetext{
* Correspondence: rholt@horizonpharma.com

${ }^{4}$ Horizon Pharma USA, Inc, 150 South Saunders Road, Lake Forest, IL 60045,

USA

${ }^{15}$ Department of Pharmacy Practice, College of Pharmacy, University of

Illinois-Chicago, 1721 North Woods Way, Vernon Hills, IL 60061, USA

Full list of author information is available at the end of the article
}

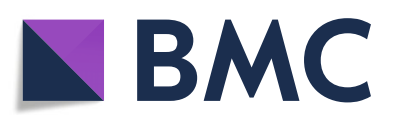

(c) The Author(s). 2018 Open Access This article is distributed under the terms of the Creative Commons Attribution 4.0 International License (http://creativecommons.org/licenses/by/4.0/), which permits unrestricted use, distribution, and reproduction in any medium, provided you give appropriate credit to the original author(s) and the source, provide a link to the Creative Commons license, and indicate if changes were made. The Creative Commons Public Domain Dedication waiver (http://creativecommons.org/publicdomain/zero/1.0/) applies to the data made available in this article, unless otherwise stated. 
(Continued from previous page)

Conclusion: NAP/ESO was well tolerated in JIA patients aged 12 to 16 years with high levels of response to ACR criteria. No new safety signals were identified for the well-characterized components of this fixed dosed JIA treatment, which was developed to reduce the risk of gastric ulcers.

Trial registration: Clinicaltrials.gov, NCT01544114. Registered February 21, 2012.

Keywords: Juvenile idiopathic arthritis, Non-steroidal anti-inflammatory drugs (NSAIDs), Naproxen, Esomeprazole

\section{Background}

Juvenile idiopathic arthritis (JIA) is an inflammatory arthritis of unknown etiology, which lasts for greater than 6 weeks with onset before 16 years of age $[1,2]$. Per the American College of Rheumatology (ACR) guidelines, JIA is the most common chronic rheumatic disease in children [3]. Non-steroidal anti-inflammatory drugs (NSAIDs) have been the mainstay of initial management with naproxen being frequently prescribed, but they can cause serious side effects such as gastric ulcers. The ACR recommends NSAIDs and/or intra-articular steroids as first line therapy for oligoarticular JIA and as supplemental to other primary therapies [3, 4]. Monotherapy has been suggested to be successful in children less than 8 years of age, with low levels of disease burden [5]. However, children of all ages generally tolerate NSAIDs better than adults, but naproxen's use in JIA is associated with gastrointestinal (GI) events in over $36 \%$ of patients, which can be a limiting factor for its use $[6,7]$. Naproxen is approved by the Food and Drug Administration (FDA) for pediatric patients $\geq 2$ years of age with JIA in dosages not greater than $15 \mathrm{mg} / \mathrm{kg} /$ day.

For pediatric patients up to 17 years of age, a delayed-release form of esomeprazole magnesium is approved in many countries for the short-term treatment (8 weeks) of gastroesophageal reflux disease and has been shown to be safe and effective [8]. An immediate-release (IR) form of esomeprazole magnesium was developed to allow for sequential gastric release of esomeprazole just prior to that of naproxen to provide maximum gastroprotective effects. A fixed combination product of this IR esomeprazole magnesium and naproxen was then developed and approved for use in adults with rheumatoid arthritis, osteoarthritis, and ankylosing spondylitis, and in adolescents with JIA, to reduce the incidence of NSAID related gastric ulcers. Herein, we report the first results of a JIA clinical efficacy and safety trial of three different doses of a combination product of naproxen with $20 \mathrm{mg}$ of IR esomeprazole.

\section{Methods}

\section{Study design}

The study was a phase 4, US only, multicenter, open-label, single arm, non-comparator study (Fig. 1) designed to evaluate the safety of $250 \mathrm{mg}, 375 \mathrm{mg}$, or $500 \mathrm{mg}$ of naproxen combined with $20 \mathrm{mg}$ of esomeprazole in a fixed combination (NAP/ESO) given twice a day (BID) $30 \mathrm{~min}$ prior to the morning and evening meals for up to 6 months.

Adolescent male and female patients, age 12 to 16 years (inclusive), with an established diagnosis of JIA as defined by the International League of Associations for Rheumatology criteria were eligible for enrollment [1]. Patients who turned 17 years of age during the study were allowed to continue in the study. Enrollment of a sufficient number of patients to obtain 45 evaluable patients at approximately 20 study sites was planned, with evaluable patients defined as those who took at least 1 dose of study drug.

The Screening period was up to 30 days, and the Treatment period with open-label NAP/ESO was up to 6 months with a 2-week follow-up period for assessment of safety, for a total of 'in study' time of 7.5 months. Patients were screened for eligibility during Visit 1 (Screening visit) after the signed informed consent and assent were obtained. Medical history and prior/concomitant medication history were obtained, a complete physical examination (blood pressure [BP], pulse rate, oral temperature, height and weight), 12-lead electrocardiogram (ECG), eye exams, and blood and urine samples for determination of clinical chemistry (including serum iron/ total iron binding capacity, vitamin B12 and magnesium), hematology and urinalysis were collected at Screening.

Screening (Visit 1) and entry into the Treatment period (Visit 2, Day 1) could have occurred during the same visit if the site performed all study Visit 1 and Visit 2 procedures within the same day, and if laboratory results were available to assess a patient's eligibility during that visit. Patients were dispensed study drug only after all Visit 1 and Visit 2 procedures were performed and eligibility was confirmed. Patients returned to the study site for scheduled visits at the end of Months 1, 3, and 6 ( \pm 7 days), and were contacted via scheduled telephone calls at Months 2, 4, and 5 ( \pm 7 days). After the end of the Treatment period, safety was assessed by telephone after a 2 -week follow-up period.

Safety assessments (adverse events [AEs], serious AEs [SAEs], concomitant medications, physical examinations with vital signs, weight, clinical chemistry and hematology) were performed at each scheduled visit. Ophthalmologic examinations were performed in accordance with the American Academy of Pediatrics guidelines [9]. AEs, SAEs, 


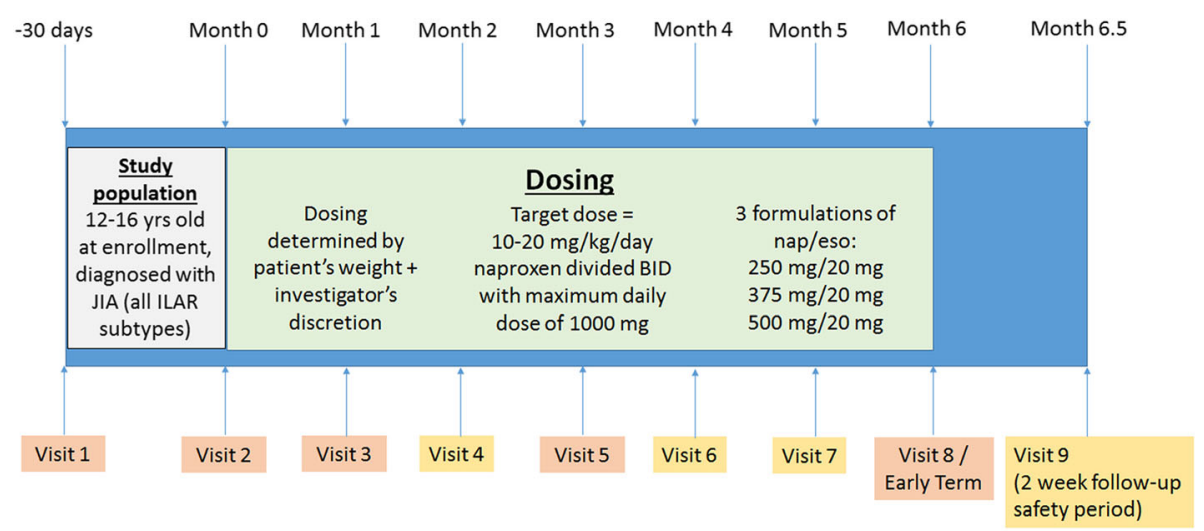

Telephone Call Study Center

Fig. 1 Overview of Study Design. Visits 1 and 2 could have been combined if results from all assessments at Visit 1 were obtained at the time of Visit 1. If Visits 1 and 2 were conducted on the same day, visit procedures that were specific to Visit 2 were also conducted during Visit 1. The telephone call during the 2-week safety follow-up period was required for all patients (i.e., patients who completed the full 6 months of treatment, patients who completed less than 6 months of treatment, patients who discontinued early from the study, and patients who took at least 1 dose of study drug)

and concomitant medications were also assessed during telephone calls. Efficacy assessments were collected at baseline and at Months 1, 3, and 6. Blood samples for naproxen and esomeprazole PK analysis were collected for frequent sampling (Month 1, up to 8 patients) or sparse sampling (Months 1 and 3, remaining patients).

Study drug was dispensed at the start of the Treatment period and at Months 1 and 3, and unused study drug was collected at Months 1, 3, and 6. The NAP/ESO strength for each patient was determined by the patient's weight at baseline (Table 1) and the Investigator's discretion according to clinical guidelines on naproxen's use [10]. The same dose assigned at baseline was maintained throughout the study. The naproxen and esomeprazole dosages are consistent with the previously identified safe and effective weight/age-appropriate doses administered to adolescent patients $[8,10,11]$. Study drug compliance was monitored at each visit by tablet counts from the returned bottles. Total tablets taken were defined as the difference of the returned from the total number dispensed. Any patient taking less than $80 \%$ or more than

Table 1 Minimum and Maximum Study Drug Dose (naproxen/ esomeprazole magnesium) by Weight Group

\begin{tabular}{lll}
\hline Weight at Enrollment $(\mathrm{kg})^{\mathrm{a}}$ & Minimum Dose $^{\mathrm{b}}$ & Maximum Dose $^{\mathrm{b}}$ \\
\hline$<38$ & $250 \mathrm{mg} / 20 \mathrm{mg}$ & $250 \mathrm{mg} / 20 \mathrm{mg}$ \\
$38-<50$ & $250 \mathrm{mg} / 20 \mathrm{mg}$ & $375 \mathrm{mg} / 20 \mathrm{mg}$ \\
$50-<75$ & $375 \mathrm{mg} / 20 \mathrm{mg}$ & $500 \mathrm{mg} / 20 \mathrm{mg}$ \\
$\geq 75$ & $500 \mathrm{mg} / 20 \mathrm{mg}$ & $500 \mathrm{mg} / 20 \mathrm{mg}$ \\
\hline
\end{tabular}

${ }^{a}$ Based on typial day-to-day fluctuations in body weight, $a \pm 3 \%$ window for body weight was permitted and used at the discretion of the Investigator when assigning the initial dose group

${ }^{\mathrm{b}}$ Study drug (naproxen/esomeprazole magnesium) dose, twice daily (BID)
$120 \%$ of the assigned study drug was considered non-compliant.

\section{Main inclusion criteria (full criteria provided in supplement)}

1. Patients were male or female adolescents age 12 to 16 years at the time of enrollment.

2. Patient was diagnosed with JIA, including all the ILAR JIA subtypes: oligoarthritis, polyarthritis (both $\mathrm{RF}+$ and RF-), psoriatic arthritis, enthesitis-related arthritis, undifferentiated arthritis, and systemic arthritis (those with presence of fever, rheumatoid rash, serositis, lymphadenopathy, macrophage activation syndrome in the past 6 months were excluded).

3. Patients who do not require ad hoc use of either active ingredient (naproxen or esomeprazole)

4. Patient's body weight was $>31 \mathrm{~kg}$ and within the 5 th to 95 th percentile of body mass index (BMI) for age.

\section{Main exclusion criteria (full criteria listed in supplement)}

1. Received treatment with any investigational agent 12 weeks or 5 half-lives of the investigational drug (whichever was longer) prior to Visit 2.

2. Had systemic JIA with presence of fever, rheumatoid rash, serositis, lymphadenopathy, macrophage activation syndrome within 6 months prior to start of study treatment.

3. Were receiving current treatment (i.e., within 4 weeks prior to start of study treatment) with 
naproxen $>20 \mathrm{mg} / \mathrm{kg} /$ day or $>1000 \mathrm{mg}$ total daily dose.

4. Had any significant unstable hepatic, renal, pulmonary, ophthalmologic, neurologic or any other medical conditions indicated by medical/ surgical history, physical or laboratory examination which would have confounded the study or put the patient at risk.

5. Had uncontrolled hypertension [12].

\section{Prior and Concomitant Therapy}

Use of the following drugs was allowed during the study:

- Concomitant JIA medications, including antitumor necrosis factors, as long as the dosing regimen was stable for 1 month prior to enrollment.

- Acetaminophen and glucocorticoid intra-articular injections on an as needed basis.

- Corticosteroids, limited to $\leq 10 \mathrm{mg}$ or $0.2 \mathrm{mg} / \mathrm{kg}$ prednisone equivalent per day (whichever was less).

- Methotrexate treatment $\leq 25 \mathrm{mg} /$ week or $15 \mathrm{mg} / \mathrm{m}^{2} /$ week (whichever was less).

Use of the following drugs was prohibited during the study:

- Treatment with another NSAID including additional naproxen at enrollment and during the study. NSAIDs, other than the study drug were prohibited during the study.

- Continuous treatment with antacids, H2-receptor antagonists, or proton pump inhibitor (PPI), in addition to the NAP/ESO received during this study.

- Continuous treatment with antifungals, antiretroviral drugs (atazanavir, nelfinavir, saquinavir), cilostazol, or warfarin (Coumadin ${ }^{\circ}$ ) or the use of these agents at any time between Visit 1 (Screening visit) and the final study visit (Visit 8 or ET visit). Concomitant use of the listed drugs is not recommended with esomeprazole.

Other medications, including over-the-counter medications and non-prescription dietary supplements that were considered necessary for the patient's safety and well-being, were permitted at the discretion of the Investigator. Patients who were on prohibited medications could participate in the study if they discontinued their medication within $24 \mathrm{~h}$ of Visit 2, if deemed medically appropriate. In addition to the prohibited/restricted medications, addition of new treatment, treatment discontinuation, and change of dosage/administration was to be avoided as much as possible.

\section{Primary study endpoints}

The primary objective was to evaluate the safety and tolerability of NAP/ESO in adolescents aged 12 to 16 years inclusive, with JIA, including the incidence and severity of adverse events (AEs) and serious AEs (SAEs), a change from baseline in vital signs, physical examination results and clinical laboratory results as well as serum iron/TIBC, vitamin B12 and magnesium levels.

Drug discontinuations were monitored monthly.

\section{Secondary objective}

The pharmacokinetic (PK) characteristics of NAP/ESO in adolescents aged 12 to 16 years, inclusive, with JIA were evaluated and compared to historically reported adult and pediatric PK data of the individual components. Overall exposure was calculated for esomeprazole and exposure to naproxen was described by an analysis of trough levels, since naproxen is slowly absorbed and excreted over time. PK parameters were compared with that published previously for adult and pediatric patients.

\section{Exploratory efficacy objective}

Disease activity was assessed with the ACR Pediatric response scores derived from the following:

- Physician's global assessment of disease activity during the last $24 \mathrm{~h}$ prior to a visit was recorded using a 21-numbered circle visual analog scale (VAS) in 0.5 increments anchored by $0=$ "no activity" and $10=$ "maximum activity." The change from baseline and percentage change from baseline was calculated at each scheduled post-baseline visit.

- CHAQ global assessment of well-being was rated for each patient by their parent at each scheduled visit using a 21-numbered VAS in 0.5 unit increments anchored by $0=$ "very well" and $10=$ "very poor" [13].

- CHAQ disability index score was calculated at each scheduled visit based on a 4-point Likert scale of $0=$ "without any difficulty," 1 = "with some difficulty," 2 = "with much difficulty", and 3 = "unable to do" as the mean of the non-missing functional area scores. If more than 2 of the 8 functional area scores were missing, then the disability index score was considered missing (i.e., a minimum of 6 functional area scores had to be non-missing).

- Number of joints with active arthritis was recorded. A joint was considered to have active arthritis if the patient had any of the following for the joint:

- Swelling, and/or

- Loss of motion and pain on motion, and/or

- Loss of motion and tenderness 
Right and left joints were considered separately (e.g., both right and left wrists could have had active arthritis and would be counted as 2 joints with active arthritis).

- Number of joints with limited range of motion was recorded. A joint was considered to have limited range of motion if the patient has any of the following for that joint:

- Loss of motion and pain on motion, and/or

- Loss of motion and tenderness.

Right and left joints were considered separately (e.g., both right and left wrists could have had limited range of motion and would be counted as 2 joints with limited range of motion).

-Either serum C-reactive protein (CRP) or erythrocyte sedimentation rate (ESR). The test done at baseline was to have been continued at post-baseline visits, to assess change from baseline.

CHAQ discomfort index was also rated for each patient by their parent at each scheduled visit using a 21-numbered VAS in 0.5 unit increments anchored by 0 = "no pain" to $10=$ "very severe pain."

CHAQ Functional area scores. Eight functional areas were assessed (dressing and grooming, arising, eating, walking, hygiene, reach, grip, and activities) on a Likert scale of 1 to 4 . For analysis purposes, responses were recoded using a scoring system of $0=$ "without any difficulty," 1 = "with some difficulty," 2 = "with much difficulty" and $3=$ "unable to do" to be consistent with the CHAQ scoring defined by Singh et al. [14].

\section{Analyses}

As this was an open-label study, no hypothesis testing was performed. All safety and efficacy endpoints were summarized by descriptive statistics. For categorical variables, counts and percentages were presented. For continuous variables, the following were presented: $n$, mean, median, standard deviation, minimum, and maximum. Change from baseline values were calculated as the visit value minus the baseline value. Negative mean numbers of the individual changes indicated an improvement from baseline.

For the PK analysis, summary statistics of trough naproxen plasma concentration (lowest plasma concentration from pre-dose to $3 \mathrm{~h}$ post-dose) during steady state were presented by dose group and visit. Empirical Bayes' estimates of the individual PK parameters were generated based on the final structural and variance parameter estimates and the individual plasma concentration measurements using nonlinear mixed effects modeling (NONMEM). Esomeprazole PK parameter estimates derived from this Population (Pop) PK analysis were analyzed and listed by study dose group and individual patient. The geometric \%CV was calculated as $100 \times \sqrt{ }\left[\exp \left(\mathrm{s}^{2}\right)-1\right]$, where $\mathrm{s}$ was the standard deviation of the data on a natural log scale.

\section{Results}

\section{Disposition and demographics}

Fifty-one patients were enrolled at 18 US sites over 2 years. Forty-six patients were assigned treatment. Forty-four patients received at least 1 dose of study drug and had at least 1 post-baseline assessment of any of the study parameters. Thirty-six $(78.3 \%)$ of the treated patients received 6 months of study drug (defined as $\geq 166$ days on study drug). Mean drug exposure was 5.5 months. See patient disposition in Table 2. Mean age, weight, and number of active joints at baseline were 13.6 years, $55.2 \mathrm{~kg}$ and 3.1, respectively (Table 3).

Five patients received glucocorticoids for JIA or "arthritis" related reasons during the study. Three patients received a total of 4 steroid joint injections. Two of the patients receiving injections also received low dose $(\leq$ $10 \mathrm{mg}$ ) oral prednisone (one for only one day and the other on numerous days), 1 received oral prednisone from 5 to $80 \mathrm{mg}$ on multiple days, and 1 received $7.5 \mathrm{mg}$ oral prednisone on one day. Of the concomitant disease modifying agents of interest, 26 (56.5\%) patients were on 1 or more of the following immunomodulators: methotrexate, adalimumab, infliximab, tocilizumab, abatacept, etanercept, leflunomide, rituximab, sulfasalazine, hydroxychloroquine sulfate. During the study, 11 patients either started new, switched immunomodulators or had their dose changed.

\section{Primary outcomes}

- Thirty-seven patients (80.4\%) had at least 1 TEAE (Table 4), as defined by the Medical Dictionary for Regulatory Activities (MedDRA). Frequent TEAEs $(\geq 5 \%)$ were upper respiratory tract infection, upper abdominal pain, sinusitis, diarrhea, headache, nausea, and ligament sprain. Four patients (8.7\%) discontinued due to a TEAE (1 SAE of hepatitis and 3 non-serious events; 1 patient with numbness, 1 patient with abdominal pain/dyspepsia, and 1 patient with worsening of JIA).

- All of the TEAEs were mild or moderate, with the exception of a 13 year old female, who had a SAE of acute hepatitis and a severe non-serious event of abnormal liver function tests. The patient had a history of hepatic steatosis (by liver biopsy), abdominal discomfort, parotitis, and jaw pain. Notably, the patient was not on concomitant methotrexate or other immunomodulators. On Day 22 of study drug treatment, the patient experienced dramatically increased AST and ALT compared to baseline. On Day 85, 
Table 2 Patient Disposition

\begin{tabular}{|c|c|c|c|c|}
\hline & & Number (\%) of $\mathrm{P}$ & & \\
\hline & $\begin{array}{l}\text { Naproxen/Esomeprazole } \\
\text { Magnesium } \\
250 \mathrm{mg} / 20 \mathrm{mg}\end{array}$ & $\begin{array}{l}\text { Naproxen/Esomeprazole } \\
\text { Magnesium } \\
375 \text { mg/20 mg }\end{array}$ & $\begin{array}{l}\text { Naproxen/Esomeprazole } \\
\text { Magnesium } \\
500 \mathrm{mg} / 20 \mathrm{mg}\end{array}$ & Total \\
\hline Enrolled & & & & 51 \\
\hline $\begin{array}{l}\text { Not assigned treatment (eligibility criteria } \\
\text { not fulfilled) }\end{array}$ & & & & 5 \\
\hline Assigned treatment & 4 & 20 & 22 & 46 \\
\hline -Received study drug & $4(100)$ & $20(100)$ & $22(100)$ & $\begin{array}{l}46 \\
(100)\end{array}$ \\
\hline $\begin{array}{l}\text {-Completed study and received } 6 \text { mo of } \\
\text { study drug }\end{array}$ & $3(75.0)$ & $16(80.0)$ & $17(77.3)$ & $\begin{array}{l}36 \\
(78.3)\end{array}$ \\
\hline -Discontinued prematurely & $1(25.0)$ & $4(20.0)$ & $5(22.7)$ & $\begin{array}{l}10 \\
(21.7)\end{array}$ \\
\hline -Adverse event & $1(25.0)$ & $1(5.0)$ & $2(9.1)$ & $4(8.7)$ \\
\hline -Lost to follow-up & 0 & $2(10.0)$ & 0 & $2(4.3)$ \\
\hline -Severe non-compliance with protocol & 0 & $1(5.0)$ & 0 & $1(2.2)$ \\
\hline -Withdrew consent & 0 & 0 & $3(13.6)$ & $3(6.5)$ \\
\hline
\end{tabular}

serologic testing was non-reactive for hepatitis A, B, and C. Study drug was permanently discontinued on Day 88 and the patient was withdrawn from the study. After withdrawal, the patient was hospitalized for a liver biopsy, which showed mixed inflammatory changes involving both hepatocytes and bile ducts in portal triads. A diagnosis of acute hepatitis with a possible etiology of drug induced or biliary cirrhosis was given. Patient was further treated in hospital and recovered. Both events were considered resolved on Day 157, with the hepatitis considered reasonably related to the study drug. Gastrointestinal AEs were reported in 17 patients, most frequently upper abdominal pain, diarrhea and nausea. (Table 4). There were no severe or serious GI events. Of these 17 patients, 7 were on concomitant disease modifying agents (methotrexate, infliximab, leflunomide and tocilizumab).

- A total of 11 (23.9\%) patients had at least 1 TEAE considered by the Investigator to be related to the study drug and 3 discontinued due to TEAE considered to be related to the study drug (hepatitis, numbness, and abdominal pain/ dyspepsia).

- The only study drug-related TEAE that occurred in $\geq 5 \%$ of all patients was upper abdominal pain (3 [6.5\%] patients).

- Due to a theoretical risk of decreased levels for iron, vitamin B12, and magnesium during long-term PPI exposure, potential alterations in these moieties during 6 months of PPI therapy were evaluated and no significant changes were seen.

\section{Secondary outcomes- pharmacokinetics}

Forty patients supplied samples for esomeprazole and 41 provided samples for naproxen. Esomeprazole concentrations observed in adolescents are comparable to those previously reported in adult and adolescent studies [15]. Geometric mean values of esomeprazole CL/F and V/F were higher in this pediatric study compared to the estimates in adult (CL/F: 24.66 vs. $12.79 \mathrm{~L} / \mathrm{h} / 70 \mathrm{~kg}^{0.75}$; V/F: 38.68 vs. $21.03 \mathrm{~L} / 70 \mathrm{~kg}$ ). Individual oral $\mathrm{CL} / \mathrm{F}$ estimates in adolescent subjects (ranging from 3.84 to $131.91 \mathrm{~L} / \mathrm{h} /$ $70 \mathrm{~kg}^{0.75}$ ) were within the range of estimates observed in adults (3.43-136.62 L/h/70 $\mathrm{kg}^{0.75}$ ) [15]. Total esomeprazole exposures (estimated as AUCs) ranged from 0.51 to $19.69 \mu \mathrm{mol} \cdot \mathrm{h} / \mathrm{L}$ in adolescent subjects and from 0.46 to $14.68 \mu \mathrm{mol} \cdot \mathrm{h} / \mathrm{L}$ in adult healthy volunteers, and are comparable to previously reported values in the literature $[8,15-17]$. Naproxen $C_{\max }$ and $T_{\max }$ data in frequently sampled pediatric patients were comparable to those reported in pediatric studies $[18,19]$ and adult studies [20]. Naproxen concentrations in terms of range and variability in this study were comparable to what was previously reported in children [18]. See supplemental materials for summary of naproxen and esomeprazole plasma concentration frequent and sparse sampling groups (Additional file 1: Tables S1 and S2).

\section{Efficacy assessment}

- The percentage of patients achieving ACR Pediatric response increased over time (Fig. 2).

- The mean baseline score for physician's assessment of disease activity was 2.58 and individual mean 
Table 3 Demographics and Baseline Characteristics

\begin{tabular}{|c|c|c|c|c|}
\hline & $\begin{array}{l}\text { Naproxen/Esomeprazole } \\
\text { Magnesium } \\
250 \mathrm{mg} / 20 \mathrm{mg} \\
(\mathrm{N}=4)\end{array}$ & $\begin{array}{l}\text { Naproxen/Esomeprazole } \\
\text { Magnesium } \\
375 \mathrm{mg} / 20 \mathrm{mg} \\
(\mathrm{N}=20)\end{array}$ & $\begin{array}{l}\text { Naproxen/Esomeprazole } \\
\text { Magnesium } \\
500 \mathrm{mg} / 20 \mathrm{mg} \\
(\mathrm{N}=22)\end{array}$ & $\begin{array}{l}\text { Total } \\
(N=46)\end{array}$ \\
\hline \multicolumn{5}{|l|}{ Age (years) } \\
\hline Mean & 12.8 & 13.6 & 13.8 & 13.6 \\
\hline SD & 0.96 & 1.47 & 1.33 & 1.37 \\
\hline Median & 12.5 & 13.0 & 13.5 & 13.0 \\
\hline Min, Max & 12,14 & 12,16 & 12,16 & 12,16 \\
\hline \multicolumn{5}{|l|}{ Sex, n (\%) } \\
\hline Female & $3(75.0)$ & $15(75.0)$ & $15(68.2)$ & $33(71.7)$ \\
\hline Male & $1(25.0)$ & $5(25.0)$ & $7(31.8)$ & $13(28.3)$ \\
\hline \multicolumn{5}{|l|}{ Race, n (\%) } \\
\hline White & $4(100)$ & $15(75.0)$ & 17 (77.3) & $36(78.3)$ \\
\hline $\begin{array}{l}\text { Black or African } \\
\text { American }\end{array}$ & 0 & $3(15.0)$ & $2(9.1)$ & $5(10.9)$ \\
\hline Asian & 0 & 0 & $2(9.1)$ & $2(4.3)$ \\
\hline Other & 0 & $2(10.0)$ & $1(4.5)$ & $3(6.5)$ \\
\hline \multicolumn{5}{|l|}{ Weight (kg) } \\
\hline Mean & 39.3 & 53.8 & 59.4 & 55.2 \\
\hline SD & 2.63 & 9.37 & 8.27 & 10.07 \\
\hline Median & 38.5 & 50.5 & 58.0 & 54.5 \\
\hline Min, Max & 37,43 & 42,76 & 49,76 & 37,76 \\
\hline \multicolumn{5}{|l|}{ Height (cm) } \\
\hline Mean & 157.5 & 158.6 & 162.5 & 160.4 \\
\hline SD & 4.73 & 8.78 & 7.70 & 8.14 \\
\hline Median & 156.0 & 156.5 & 159.5 & 158.5 \\
\hline Min, Max & 154,164 & 144,175 & 152,176 & 144,176 \\
\hline \multicolumn{5}{|l|}{ BMI $\left(\mathrm{kg} / \mathrm{m}^{2}\right)$} \\
\hline Mean & 15.86 & 21.34 & 22.56 & 21.45 \\
\hline SD & 1.579 & 2.797 & 3.476 & 3.534 \\
\hline Median & 15.41 & 20.42 & 21.69 & 20.75 \\
\hline Min, Max & $14.5,18.1$ & $16.8,26.6$ & $17.3,32.0$ & $\begin{array}{l}14.5 \\
32.0\end{array}$ \\
\hline \multicolumn{5}{|c|}{ No. of Joints With Active Arthritis ${ }^{a}$} \\
\hline $\mathrm{N}$ & 4 & 19 & 21 & 44 \\
\hline Mean & 1.0 & 4.1 & 2.5 & 3.1 \\
\hline SD & 1.15 & 10.56 & 3.84 & 7.39 \\
\hline Median & 1.0 & 1.0 & 1.0 & 1.0 \\
\hline Min, Max & 0,2 & 0,46 & 0,16 & 0,46 \\
\hline
\end{tabular}

$B M I$ body mass index, max maximum, min minimum, $S D$ standard deviation

${ }^{a} A$ joint was considered to have active arthritis if the patient had any of the following: swelling, loss of motion and pain on motion, and/or loss of motion and tenderness. Right and left joints were considered separately (e.g., both right and left wrists could have had active arthritis and would have been counted as 2 joints with active arthritis)

improvement from baseline was $-0.67,-0.99$, and -1.23 at Months 1, 3, and 6, respectively.

- Mean CHAQ global assessment of Well-Being baseline score was 3.43 and mean improvement from baseline was $-0.80,-0.93$, and -1.39 at Months 1 , 3 , and 6.

- Mean CHAQ functional scores indicated improvement from baseline for 3 of the functional 
Table 4 TEAEs occurring in at least 2 patients

\begin{tabular}{|c|c|c|c|c|}
\hline \multirow[b]{2}{*}{$\begin{array}{l}\text { MedDRA System Organ Class } \\
\text { Preferred Term }\end{array}$} & \multicolumn{4}{|c|}{ Number (\%) of Patients } \\
\hline & $\begin{array}{l}\text { NAP/ESO } \\
250 \mathrm{mg} / 20 \mathrm{mg} \\
(\mathrm{N}=4)\end{array}$ & $\begin{array}{l}\text { NAP/ESO } \\
375 \mathrm{mg} / 20 \mathrm{mg} \\
(\mathrm{N}=20)\end{array}$ & $\begin{array}{l}\text { NAP/ESO } \\
500 \mathrm{mg} / 20 \mathrm{mg} \\
(\mathrm{N}=22)\end{array}$ & $\begin{array}{l}\text { Total } \\
(N=46)\end{array}$ \\
\hline Patient with any TEAE & $4(100)$ & $16(80.0)$ & $17(77.3)$ & $37(80.4)$ \\
\hline Gastrointestinal disorders & $1(25.0)$ & $8(40.0)$ & $8(36.4)$ & $17(37.0)$ \\
\hline Abdominal pain upper & 0 & $3(15.0)$ & $2(9.1)$ & $5(10.9)$ \\
\hline Diarrhea & 0 & $2(10.0$ & $2(9.1)$ & $4(8.7)$ \\
\hline Nausea & 0 & $3(15.0)$ & $1(4.5)$ & $4(8.7)$ \\
\hline Abdominal discomfort & 0 & $2(10.0)$ & 0 & $2(4.3)$ \\
\hline Dyspepsia & 0 & 0 & $2(9.1)$ & $2(4.3)$ \\
\hline Vomiting & 0 & $1(5.0)$ & $1(4.5)$ & $2(4.3)$ \\
\hline Infections and infestations & $2(50.0)$ & $4(20.0)$ & $9(40.9)$ & $15(32.6)$ \\
\hline Upper respiratory tract infection & $1(25.0)$ & $2(10.0)$ & $6(27.3)$ & $9(19.6)$ \\
\hline Sinusitis & 0 & $1(5.0)$ & $4(18.2)$ & $5(10.9)$ \\
\hline Gastroenteritis viral & 0 & 0 & $2(9.1)$ & $2(4.3)$ \\
\hline Tooth infection & 0 & $1(5.0)$ & $1(4.5)$ & $2(4.3)$ \\
\hline Musculoskeletal and connective tissue disorders & $2(50.0)$ & $2(10.0)$ & $2(9.1)$ & $6(13.0)$ \\
\hline Back pain & $1(25.0)$ & $1(5.0)$ & 0 & $2(4.3)$ \\
\hline Pain in extremity & 0 & $1(5.0)$ & $1(4.5)$ & $2(4.3)$ \\
\hline Injury, poisoning and procedural complications & 0 & $2(10.0)$ & $3(13.6)$ & $5(10.9)$ \\
\hline Ligament sprain & 0 & 0 & $3(13.6)$ & $3(6.5)$ \\
\hline Nervous system disorders & $2(50.0)$ & $1(5.0)$ & $2(9.1)$ & $5(10.9)$ \\
\hline Headache & $1(25.0)$ & $1(5.0)$ & $2(9.1)$ & $4(8.7)$ \\
\hline General disorders and administration site conditions & 0 & 0 & $2(9.1)$ & $2(4.3)$ \\
\hline Fatigue & 0 & 0 & $2(9.1)$ & $2(4.3)$ \\
\hline Immune system disorders & 0 & $1(5.0)$ & $1(4.5)$ & $2(4.3)$ \\
\hline Hypersensitivity & 0 & $1(5.0)$ & $1(4.5)$ & $2(4.3)$ \\
\hline Neoplasms benign, malignant and unspecified (incl. cysts and polyps) & $1(25.0)$ & $1(5.0)$ & 0 & $2(4.3)$ \\
\hline Skin papilloma & $1(25.0)$ & $1(5.0)$ & 0 & $2(4.3)$ \\
\hline Respiratory, thoracic and mediastinal disorders & 0 & $1(5.0)$ & $1(4.5)$ & $2(4.3)$ \\
\hline Cough & 0 & $1(5.0)$ & $1(4.5)$ & $2(4.3)$ \\
\hline
\end{tabular}

areas (arising, walking, and activities). For 4 of the functional areas (dressing and grooming, eating, hygiene, and grip), the mean change from baseline indicated an improvement or no change (mean change 0.0) at each assessment. For the remaining functional area of 'reach', there was no change at each post-baseline assessment except Month 1 (mean change 0.2).

- The mean baseline score for the CHAQ disability index was 0.506. The mean improvement from baseline was $-0.069,-0.064$, and -0.155 at Months 1,3 , and 6 , respectively.

- The mean baseline number of joints with active arthritis was 3.1. The individual mean change indicated an improvement from baseline to each post-baseline assessment. The mean improvement from baseline was $-1.1,-0.4$, and -0.6 at Months 1,3 , and 6 , respectively.

- The mean baseline number of joints with limited range of motion was 1.7 . The individual mean improvement from baseline was $-0.9,-0.1$, and 0.2 at Months 1, 3, and 6, respectively.

- The mean baseline serum CRP was $8.807 \mathrm{mg} / \mathrm{mL}$ with a mean change of $0.169,-1.076$, and -1.120 at Months 1, 3, and 6, respectively, indicating individual mean improvement at Months 3 and 6 .

- The mean baseline serum ESR was $10.4 \mathrm{~mm} / \mathrm{hr}$. The mean individual change indicated improvement 

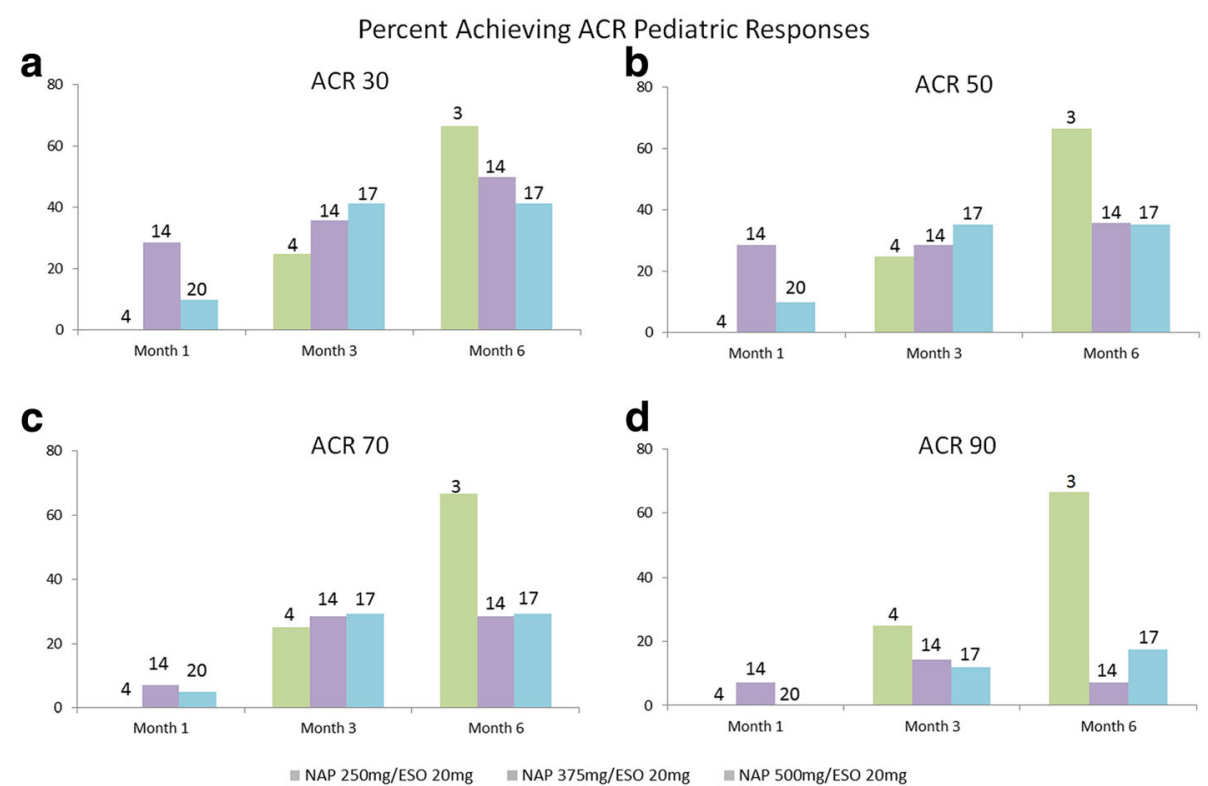

Fig. 2 ACR Scores. The number above each bar represents the number of patients at that dose. The four patients in the lowest dose group did not reach any ACR response at Month 1. The ACR Pediatric-30, $-50,-70$, and -90 responses were defined as an improvement of at least 30\% (or $50,70,90 \%$, respectively) from baseline in at least 3 of the 6 signs and symptoms variables, with no more than 1 of the remaining variables worsening by $>30 \%$. JIA signs and symptoms variables: physician's global assessment of disease activity, CHAQ disability index score, CHAQ global assessment of well-being, number of joints with active arthritis, number of joints with limited range of motion, serum CRP or ESR

from baseline to Month 3. The mean change from baseline was $0.4,-0.1$, and 0.8 at Months 1,3 , and 6 , respectively.

- CHAQ discomfort improved at each assessment from a baseline mean of 4.41. The mean improvement from baseline was $-1.24,-1.21$, and -2.20 at Months 1, 3, and 6.

- There was no indication of a dose-related efficacy effect in any of the outcomes.

- Adherence with study medication was good with $73.9 \%$ of patients ( 34 of 46 ) with $\geq 80 \%$ and $\leq 120 \%$ compliance and $21.7 \%$ (10 of 46 patients) demonstrating $<80 \%$ or $>120 \%$ (non-compliance). Two subjects were lost to follow-up and therefore did not have an overall compliance calculated.

\section{Additional analyses}

Twenty-six of the 46 patients who were assigned treatment with NAP/ESO were also taking a DMARD/biologic during the study and the remaining patients utilized NAP/ESO as their primary therapy. Those who were on co-therapy and had variables to determine a month 6 ACR response $(N=21)$ displayed a greater increase in percentage of patients with ACR Response 30, $-50,-70$, and -90 when compared to those using NAP/ESO as their primary therapy (Fig. 3). Baseline demographics of the co-therapy group appeared similar to the primary therapy group (see Additional file 1: Table S3), and baseline disease measures showed a trend of increased severity in the co-therapy group (see Additional file 1: Table S4).

\section{Discussion}

The fixed combination of naproxen and esomeprazole was well tolerated in this population of 12 to 16 -year-old patients with JIA, and no new safety signals were

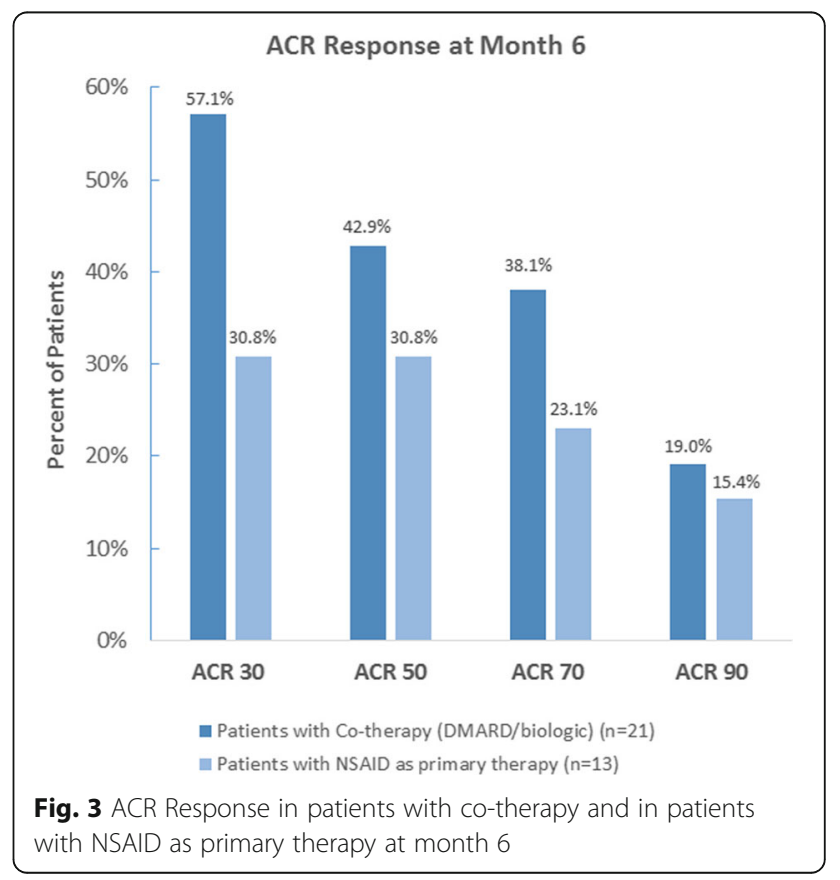


identified. The safety results were consistent with the well-characterized profiles of the components; naproxen and esomeprazole magnesium.

Esomeprazole was selected as the PPI of choice for this combination because of its well-documented acid-inhibiting properties [21] and its proven efficacy in reducing the risk of NSAID-associated gastric ulcers [22]. Naproxen was chosen because historically, up to $85 \%$ of JIA patients have been treated with NSAIDs [23], with naproxen noted to be the predominate NSAID in use recently [5]. In a previous trial, naproxen was noted to produce GI AEs in $39.6 \%$ of patients with the event rate driven predominantly by abdominal pain in an adolescent patient population aged 12-17, similar to this study. The abdominal pain rate found in this study was $10.9 \%$ [24]. Research should continue on identifying which JIA patients will benefit the most from NSAID therapy as well as those most at risk for NSAID induced GI events who would benefit from gastroprotection with this fixed combination of naproxen and esomeprazole.

Our study found good results with naproxen/esomeprazole monotherapy with ACR 30 and 50 responses in $31 \%$ of our JIA patients. However, our data seem to indicate that patients with higher disease activity respond better to combination immunomodulator therapy and naproxen/esomeprazole, as evidenced by higher ACR 30 and 50 responses, though none of the baseline differences were statistically significantly different between the two groups.

At baseline, patients in this study were on mean approximately 14 years of age, had 3 active joints, and a CRP of about 9. Our patients demonstrated good responses over the observation period with $47 \%$ achieving an ACR-30, 38\% receiving an ACR-50, 32\% receiving an ACR-70 response, and 18\% receiving an ACR-90 response at month 6 . Improvements were observed in JIA signs and symptoms, the CHAQ discomfort index, and several CHAQ functional area scores. The percentage of patients that achieved an ACR Pediatric response increased over time during the study and were generally similar to historic NSAID study results indicating significant responses between 25 to $33 \%$ of patients [25].

There was no association of the weight based doses with response indicating that the doses chosen in this study were appropriate and in line with published guidelines [10]. We did not, however, titrate doses to determine optimal efficacy, but the doses used in this study (mean of $\approx 15.5 \mathrm{mg} / \mathrm{kg} /$ day) represent previously identified effective doses of naproxen for JIA treatment, with a target range of between 10 and $20 \mathrm{mg} / \mathrm{kg} /$ day divided twice daily with a maximum of $1000 \mathrm{mg} /$ day. Only 4 patients received the lowest dose $(250 \mathrm{mg} \mathrm{NAP} / 20 \mathrm{mg}$ ESO), and therefore there was limited data within the lowest weight group, leading to the currently recommended FDA approval of the fixed dosage form in those patients 12 years of age and older weighing at least $38 \mathrm{~kg}$, requiring naproxen for symptomatic relief of arthritis and esomeprazole magnesium to decrease the risk of developing naproxen-associated gastric ulcers [26].

Mean drug exposure was approximately 165 days in the 46 patients, with $36(78.3 \%)$ of them receiving 6 months of study drug (defined as $\geq 166$ days on study drug). The PK results approximated the esomeprazole PK data from previously published pediatric studies. The naproxen $C_{\max }$ and $T_{\max }$ values in the more frequently sampled pediatric patients were comparable to those reported in the literature [18-20]. In addition, naproxen concentrations in terms of range and variability in this pediatric study were comparable to that previously reported in children. These results indicated that combining esomeprazole and naproxen did not result in any significant interaction affecting overall pharmacokinetics of the individual components of the fixed dosage form.

\section{Conclusions}

The fixed combination of NAP/ESO was well tolerated in JIA patients aged 12 to 16 years. No new safety signals were identified for the well-characterized components of this fixed dosed JIA treatment, which was developed to reduce the risk of gastric ulcers in patients requiring chronic naproxen therapy. Improvement in JIA signs and symptoms occurred at most assessments and by month 6, the percentage of patients with an ACR Pediatric-30, - 50, -70, and-90 Response was 47.1, $38.2,32.4$, and $17.6 \%$, respectively.

\section{Additional file}

Additional file 1: Supplemental Data. (DOCX $54 \mathrm{~kb}$ )

\section{Acknowledgements}

The authors would like to thank the following study site coordinators and the patients for their contributions to this study: Catherine Calacanis, Chidima Martin loanou, Debra Latham, Bob Chaney, Janette Groth, Marilyn Orlando, Nicole Battle, Kym Abraham, Nanastasia Welnik, Manju Gupta, Heidi Stapp, Mignon Hills, Marsha Simmons, Kathy Hummel, and Christy Reed. In addition, the authors would like to thank Teresa M.Y. Kok RPh, MBA from Horizon Pharma for data analysis, writing and editorial support.

\section{Funding}

Horizon Pharma USA, Inc. funded the development, conduct, and analysis of the study.

\section{Availability of data and materials}

The data sets generated during the current study are not publicly available but may be available from the corresponding author. Data are also available on clinicaltrials.gov (NCT01544114).

\section{Authors' contributions}

All named authors meet the International Committee of Medical Journal Editors (ICMJE) criteria for authorship for this manuscript, accept responsibility for the integrity of the work as a whole, and have provided final approval of the manuscript to be published. 


\section{Ethics approval and consent to participate}

An Institutional Review Board (IRB)/Ethics Committee (EC) from each clinical study site approved the final study protocol, and the final version of the informed consent form and assent form and any other written information and/or materials provided to the patients before any patients were entered in the study. Before being implemented, protocol amendments were reviewed and approved by the IRBs.

\section{Consent for publication}

Not applicable.

\section{Competing interests}

D.J.L. receives grant / research support from the National Institutes of Health, NIAMS; Cincinnati Children's Hospital Medical Center receives funds for DJL consulting from AstraZeneca, Bristol-Myers Squibb, AbbVie, Pfizer, Roche, Novartis, UBC, Forest Research Institute, Horizon, Johnson \& Johnson, Biogen, Takeda, Genentech, GlaxoSmithKline, Boehringer Ingelheim, Celgene, and Janssen for consulting; He is on the speaker bureaus of Genentech and Bristol Meyers Squibb. J.A.D. has received research support from AbbVie, AstraZeneca, Bristol-Myers Squibb, Horizon Pharma, Medac, Pfizer, Roche and UCB. D.G. is on Novartis speaker bureau. L.J. has received research support from Abbvie, GSk, Hoffman-Laroche, Pfizer and UCB. A.Z. has stock ownership in Merck and OPKO. J.B., B.D.L., M. F. and R.J. H. are employees of, and have stock in, Horizon Pharma USA, Inc.

\section{Publisher's Note}

Springer Nature remains neutral with regard to jurisdictional claims in published maps and institutional affiliations.

\section{Author details}

'Cincinnati Children's Hospital Medical Center, 3333 Burnet Ave, Cincinnati, $\mathrm{OH} 45229$, USA. ${ }^{2}$ University of Cincinnati School of Medicine, 3230 Eden Ave, Cincinnati, OH 45267, USA. ${ }^{3}$ Arkansas Children's Hospital, 1 Children's Way, Slot\# 512-2, Little Rock, AR 72202, USA. ${ }^{4}$ Horizon Pharma USA, Inc, 150 South Saunders Road, Lake Forest, IL 60045, USA. ${ }^{5}$ University of California San Francisco Pediatric Rheumatology, 550 16th Street, 5th Fl, San Francisco, CA 94158, USA. 'University of Nebraska Medical Center/Children's Hospital and Medical Center, 8200 Dodge St, Omaha, NE 68114, USA. ${ }^{7}$ Augusta University Medical Center, 1120 15th Street, Augusta, GA 30912-5536, USA. ${ }^{8}$ O \& O Alpan, LLC, 11212 Waples Mill Rd Ste. 100, Fairfax, VA 22030, USA. ${ }^{9}$ Rheumatology and Immunology Private Practice, 6005 Park Ave, Suite 409, Memphis, TN 38119, USA. ${ }^{10}$ St. Christopher's Hospital for Children, 160 E Erie Ave, Philadelphia, PA 19134, USA. ${ }^{11} T$ The Cleveland Clinic, 9500 Euclid Avenue, Cleveland, OH 44195, USA. ${ }^{12}$ Toledo Clinic Inc, 4235 Secor Road, Toledo, OH 43623, USA. ${ }^{13}$ Cohen Children's Medical Center of New York, 269-01 76th Avenue, New Hyde Park, NY 11040, USA. ${ }^{14}$ Children's National Medical Center, 111 Michigan Avenue, NW, Washington, DC 20010, USA. ${ }^{15}$ Department of Pharmacy Practice, College of Pharmacy, University of Illinois-Chicago, 1721 North Woods Way, Vernon Hills, IL 60061, USA.

Received: 29 May 2018 Accepted: 14 June 2018

Published online: 26 June 2018

\section{References}

1. Petty RE, Southwood TR, Manners P, Baum J, Glass DN, Goldenberg J, et al. International league of associations for rheumatology classification of juvenile idiopathic arthritis: second revision, Edmonton, 2001. J Rheumatol. 2004;31(2):390-2.

2. Rigante D, Bosco A, Esposito $S$. The etiology of juvenile idiopathic arthritis. Clin Rev Allergy Immunol. 2015;49(2):253-61.

3. Ringold S, Weiss PF, Beukelman T, DeWitt EM, llowite NT, Kimura Y, et al. 2013 update of the 2011 American College of Rheumatology recommendations for the treatment of juvenile idiopathic arthritis: recommendations for the medical therapy of children with systemic juvenile idiopathic arthritis and tuberculosis screening among children receiving biologic medications. Arthritis Rheum 2013;65(10):2499-2512.

4. Beukelman T, Patkar NM, Saag KG, Tolleson-Rinehart S, Cron RQ, DeWitt EM, et al. 2011 American College of Rheumatology recommendations for the treatment of juvenile idiopathic arthritis: initiation and safety monitoring of therapeutic agents for the treatment of arthritis and systemic features. Arthritis care Res. 2011;63(4):465-82.
5. Sura A, Failing C, Sturza J, Stannard J, Riebschleger M. Patient characteristics associated with response to NSAID monotherapy in children with systemic juvenile idiopathic arthritis. Pediatr Rheumatol. 2018;16(1):2.

6. Cimaz R, Marino A, Martini A. How I treat juvenile idiopathic arthritis: a state of the art review. Autoimmun Rev. 2017;16(10):1008-15.

7. Foeldvari I, Szer IS, Zemel LS, Lovell DJ, Giannini EH, Robbins JL, et al. A prospective study comparing celecoxib with naproxen in children with juvenile rheumatoid arthritis. J Rheumatol. 2009;36(1):174-82.

8. Nexium [package insert]. AstraZeneca pharmaceuticals LP, Wilmington; 2016.

9. Cassidy J, Kivlin J, Lindsley C, Nocton J. Ophthalmologic examinations in children with juvenile rheumatoid arthritis. Pediatrics. 2006;117(5):1843-5.

10. Cassidy J, Petty R, Laxer R, Lindsley C. Textbook of pediatric rheumatology. 6th ed. Philadelphia: Elsevier; 2011.

11. Weiss JE, llowite NT. Juvenile idiopathic arthritis. Pediatr Clin N Am. 2005: 52(2):413-42. vi

12. National High Blood Pressure Education Program Working Group on High Blood Pressure in Children and Adolescents. The fourth report on the diagnosis, evaluation, and treatment of high blood pressure in children and adolescents. Pediatrics. 2004;114(2 Suppl 4th Report):555-76.

13. Filocamo G, Davi S, Pistorio A, Bertamino M, Ruperto N, Lattanzi B, et al. Evaluation of 21-numbered circle and 10-centimeter horizontal line visual analog scales for physician and parent subjective ratings in juvenile idiopathic arthritis. J Rheumatol. 2010;37(7):1534-41.

14. Singh G, Athreya BH, Fries JF, Goldsmith DP. Measurement of health status in children with juvenile rheumatoid arthritis. Arthritis Rheum. 1994;37(12): 1761-9.

15. VIMOVO NDA 22511 Efficacy Supplement 2017. https://www.fda.gov/ downloads/Drugs/DevelopmentApprovalProcess/DevelopmentResources/ UCM571347.pdf. Accessed 21 Jan 2018.

16. Hassan-Alin M, Andersson T, Bredberg E, Rohss K. Pharmacokinetics of esomeprazole after oral and intravenous administration of single and repeated doses to healthy subjects. Eur J Clin Pharmacol. 2000;56(910):665-70

17. Li J, Zhao J, Hamer-Maansson JE, Andersson T, Fulmer R, Illueca M, et al. Pharmacokinetic properties of esomeprazole in adolescent patients aged 12 to 17 years with symptoms of gastroesophageal reflux disease: a randomized, open-label study. Clin Ther. 2006;28(3):419-27.

18. Valitalo P, Kumpulainen E, Manner M, Kokki M, Lehtonen M, Hooker AC, et al. Plasma and cerebrospinal fluid pharmacokinetics of naproxen in children. J Clin Pharmacol. 2012;52(10):1516-26.

19. Wells TG, Mortensen ME, Dietrich A, Walson PD, Blasier D, Kearns GL. Comparison of the pharmacokinetics of naproxen tablets and suspension in children. J Clin Pharmacol. 1994:34(1):30-3.

20. Naprosyn [package insert]. Genentech, San Francisco; 2013.

21. Miner P Jr, Katz PO, Chen Y, Sostek M. Gastric acid control with esomeprazole, lansoprazole, omeprazole, pantoprazole, and rabeprazole: a five-way crossover study. Am J Gastroenterol. 2003:98(12):2616-20.

22. Scheiman JM, Yeomans ND, Talley NJ, Vakil N, Chan FK, Tulassay Z, et al. Prevention of ulcers by esomeprazole in at-risk patients using non-selective NSAIDs and COX-2 inhibitors. Am J Gastroenterol. 2006;101(4):701-10.

23. Cron RQ, Sharma S, Sherry DD. Current treatment by United States and Canadian pediatric rheumatologists. J Rheumatol. 1999;26(9):2036-8.

24. Reiff A, Lovell DJ, Adelsberg JV, Kiss MH, Goodman S, Zavaler MF, et al. Evaluation of the comparative efficacy and tolerability of rofecoxib and naproxen in children and adolescents with juvenile rheumatoid arthritis: a 12-week randomized controlled clinical trial with a 52-week open-label extension. J Rheumatol. 2006;33(5):985-95.

25. Giannini EH, Cawkwell GD. Drug treatment in children with juvenile rheumatoid arthritis. Past, present, and future. Pediatr Clin N Am. 1995;42(5): 1099-125.

26. Vimovo [package insert]. Horizon Pharma USA, Inc., Lake Forest; 2017. 